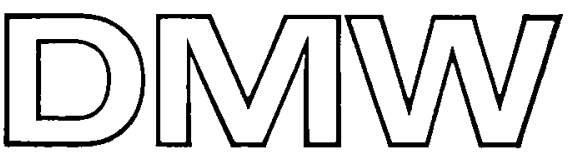

\title{
Diagnose-Verschlüsselung: wie und wozu?
}

Die neue Bundespflegesatzverordnung schreibt vor, daß jedes Krankenhaus als wesentliche Basisinformation für Pflegesatzverhandlungen eine Diagnosestatistik vorlegt. Sie beruht auf der dreistelligen Kurzfassung des ICD-9Schlüssels (Internationale Klassifikation der Krankheiten, 9. Revision) (1). $\mathrm{Zu}$ jeder der hier vorgesehenen Diagnosegruppen sollen die Anzahl der behandelten Patienten, die Verweildauer und gegebenenfalls die Anzahl der Operationen, aufgeschlüsselt nach dem Alter der Patienten, angegeben werden.

Durch die jetzt unumgängliche Verschlüsselung der Diagnosen eröffnen sich vor allem für die Forschung, aber auch für Lehre und Weiterbildung neue Möglichkeiten. Ferner können mit der zur Verfügung stehenden EDV-Kapazität weiterführende Spezialkarteien integriert werden. Um den damit verbundenen Anforderungen gerecht zu werden; müssen die Diagnosen allerdings hinreichend detailliert erfaßt werden. Auch sollten eine gute begriffliche Präzision und eine möglichst einheitliche Nomenklatur der Diagnosen gewährleistet sein. Der benutzte Schlïssel sollte in dieser Hinsicht auch »erzieherisch " auf den (ärztlichen) Anwender wirken.

Der ICD-9-Schlüssel eignet sich für diese Ziele allerdings nur bedingt. Er wurde ursprünglich zur Verschlüsselung von Todesursachen entwickelt und erst in jüngster Zeit zu einem allgemeinen Diagnoseschlüssel erweitert, wobei die im Rahmen dieser Entwicklung notwendige Anpassung an die Erfordernisse der Krankenversicherungen und der Krankenhäuser mit der 9. Revision durchaus noch nicht abgeschlossen war. Besondere Kritikpunkte sind erhebliche Abweichungen von der gängigen klinischen Nomenklatur, die Benutzung von zum Teil altertümlichen Synonymen und die mangelnde Unterscheidung von Manifestationsformen von Krankheiten und ätiologisch zugrunde liegenden Krankheitsbildern. Teilweise sind sogar Doppelverschlüsselungen zugelassen. Nicht zuletzt fehlt die Möglichkeit, Operationen und Behandlungsverfahren zu verschlüsseln. Entsprechende Zusatzklassifikationen sind in der deutschen Übersetzung nicht enthalten.

Aufgrund der offensichtlichen Mängel der ICD-9 wurde in den USA bereits 1979 eine klinische Modifikation dieses Schlüssels (ICD-9-CM: International Classification of Diseases, 9th Revision, Clinical Modification)

Dtsch. med. Wschr. 111 (1986), 1707-1708

(C) 1986 Georg Thieme Verlag Stuttgart . New York fertiggestellt. Die Krankheiten werden darin, soweit erforderlich, detaillierter erfaßt, Doppelverschlüsselungen wurden, wenn möglich, eliminiert, Symptome werden von ätiologischen Kategorien besser unterschieden, und schließlich wurde auch ein Zusatzschlüssel für Operationen und Behandlungsverfahren integriert ("Procedure Classification ").

Eine deutsche Übersetzung der ICD-9-CM liegt bisher nicht vor. Wir haben daher für die Medizinische Universität zu Lübeck mit entsprechender Zielsetzung eine eigene Modifikation des ICD-9-Schlüssels erarbeitet, die in der 1. Auflage zunächst nur wesentliche nicht-operative Fachgebiete (Innere Medizin, Psychiatrie, Neurologie, Dermatologie) betrifft. Die Berücksichtigung der operativen Fächer und der Pädiatrie erfolgte vorerst provisorisch mit unveränderten dreistelligen ICD-Nummern. Erste Erfahrungen mit dem praktischen Einsatz unseres Schlüssels sind ermutigend. Für die Zukunft versprechen wir uns aufgrund dieses Diagnoseschlüssels verbesserte Möglichkeiten sowohl für die wissenschaftliche als auch für die abrechnungsbezogene Auswertung der gesammelten Daten. Weitere Ansätze zu einer fächerübergreifenden Modifikation des ICD-9-Schlüssels sind uns aus dem deutschen Raum bisher nicht bekanntgeworden. Lediglich für die operativen Fachgebiete, die durch das Fehlen eines Operationskatalogs in der ICD-9 in besonderer Weise betroffen sind, scheint sich eine Orientierung am Operationsschlüssel der Vereinigung Schweizerischer Krankenhäuser (VESKA) abzuzeichnen.

Für die praktische Durchführung der Diagnose-Codierung gibt es zur Zeit verschiedene Konzepte. In einigen Häusern erfolgt die Verschlüsselung der Diagnosen durch den zuletzt behandelnden Arzt (zum Beispiel bei Abfassung des Arztbriefes), während in anderen die Codierung anhand »nicht genormter " Arztbriefdiagnosen durch Hilfspersonal oder durch automatische Computerprogramme (zum Beispiel DIACOS) vorgesehen ist.

Während die Codierung durch den Arzt ein hohes $\mathrm{Maß}$ an Zuverlässigkeit verspricht und hinsichtlich der Präzision durch entsprechende Vorgaben im Schlüssel steuerbar ist, ergeben sich aus einer nachträglichen Verschlüsselung nicht nur aufgrund der verbreiteten nomenklatorischen Unsicherheiten erhebliche Schwierigkeiten. Insbesondere wären einige der genannten Anforderungen damit nicht zu erfüllen. Da der Arzt hinsichtlich der Präzision seiner Diagnosestellung bei einem derartigen System völlige Freiheit hätte und keinen Vorgaben folgen 
müßte, würden in vielen Fällen Daten fehlen, die für eine sinnvolle Nutzung der Datensammlung erforderlich sind. Diese Informationen können ohne nachträgliches Aktenstudium nicht mehr ergänzt werden. Wir haben uns daher für eine Codierung seitens des behandelnden Arztes mit Kontrolle durch den Oberarzt oder Chefarzt (bei Vorlage des Arztbriefes) entschieden.

Weitere Schwierigkeiten ergeben sich bei der Falldefinition. Hier ist zum Beispiel unklar, wie vorübergehende Entlassungen mit geplanten Wiederaufnahmen, etwa kurzzeitige Entlassungen zwischen Behandlungen oder Untersuchungsterminen, oder klinikinterne Verlegungen, insbesondere von Intensivstationen, zu behandeln sind, obwohl sich hieraus erhebliche Konsequenzen für die Berechnung der Verweildauer ergeben. Durchführungsrichtlinien hierfür werden allerdings bereits erarbeitet.

Die Diagnosestatistik soll nach der Vorstellung des Gesetzgebers Leistungsvergleiche zwischen den Krankenhäusern ermöglichen. Ob eine derartige Leistungstransparenz aber tatsächlich erreicht werden kann, bleibt zu bezweifeln. Hierzu ist ein Vergleich mit dem amerikanischen Abrechnungssystem für die staatliche Krankenversicherung (Medicare) aufschlußreich, das 1983 reformiert worden ist (5). Die herkömmliche kostenbezogene Abrechnung seitens der Krankenhäuser wurde durch eine prospektiv orientierte Zahlungsweise auf der Basis vorher festgelegter Fallkostenpauschalen ersetzt. Die Bezahlung wird dabei durch die Entlassungsdiagnosen bestimmt, die einer von 467 "diagnosis-related groups " zugeordnet werden, für die vorher bestimmte, von den tatsächlich im Einzelfall angefallenen Kosten unabhängige Festpreise abgerechnet werden. Diagnosebezogene Gruppen sind Gruppen von Krankheitsbildern, die aufgrund empirischer Untersuchungen (2) weitgehend homogen hinsichtlich der Behandlungskosten sein sollen. Die Zuordnung eines Behandlungsfalles zu einer solchen Gruppe erfolgt aufgrund der Haupt- und Nebendiagnose(n) (codiert nach ICD-9-CM), des Alters des Patienten und eventueller Operationen.

Die neue Bundespflegesatzverordnung nimmt auf derartige Gruppen oder ähnliche Kategorien bisher keinen Bezug. Da die Kategorien der ICD-9 im Gegensatz zu den diagnosebezogenen Gruppen nicht unter dem Gesichtspunkt der Kostenhomogenität entwickelt wurden, eignen sie sich nicht für eine leistungsorientierte Abrechnung. So codiert beispielsweise der ICD-Code 250 sowohl einen "Diabetes mellitus ohne Angabe einer Komplikation " als auch "Diabetes mit Koma" oder "Diabetes mit Gan-

grän " usw., also verschiedene Krankheitsformen, die sicher nicht kostenhomogen sind. Eine nachträgliche Bildung von diagnosebezogenen Gruppen dürfte angesichts der unpräzisen dreistelligen Diagnose-Codierung und des völligen Fehlens einer Regelung für eine Codierung der operativen Verfahren nicht möglich sein, da die hierzu notwendigen Informationen nicht erfaßt werden.

Bedenkt man, daß bereits die weitaus genauer definierten diagnosebezogenen Gruppen in den USA hinsichtlich ihrer Kosten- und Leistungshomogenität stark umstritten sind und ihre Brauchbarkeit für Abrechnungszwecke schon aus diesem Grunde angezweifelt wird $(3,4,7,8)$, so ist zu erahnen, daß die bei uns eingeführten, lediglich dreistelligen ICD-Codes ein noch viel weniger geeignetes Verhandlungsinstrument sein werden. Abgesehen von diesen eher technischen Gesichtspunkten bleibt aber das fallbezogene Abrechnungssystem in den USA auch aus anderen Gründen, besonders auch hinsichtlich seiner kostendämpfenden Wirkung und nicht zuletzt auch hinsichtlich seiner Auswirkungen auf die Qualität der medizinischen Versorgung, umstritten $(4,9,10)$. Das sollte bei der auch in der Bundesrepublik geführten Diskussion um Kostensenkungseffekte von Fallpauschalen $(6,11)$ nicht unberücksichtigt bleiben.

\section{Literatur}

(1) Der Bundesminister für Jugend, Familie und Gesundheit (Hrsg.): Handbuch der internationalen Klassifikation der Krankheiten, Verletzungen und Todesursachen (ICD) 1979, 9. Revision (Girardet: Wuppertal 1979)

(2) Fetter, R. B., Y. Shin, J. L. Freeman, R. F. Averill, J. D Thompson: Case mix definition by diagnosis-related groups. Med. Care 18, Suppl. (1980), 1.

(3) Garber, A. M., V. R. Fuchs, J. F. Silverman: Case mix, costs, and outcomes. New Engl. J. Med. 310 (1984), 1231.

(4) Horn, S. D., R. A. Horn, P. D. Sharkey, R. J. Beall, J. S. Hoff, B. J. Rosenstein: Misclassification problems in diag nosis-related groups. New Engl. J. Med. 314 (1986), 484.

(5) Iglehart, J. K.: Medicare begins prospective payment of hospitals. New Engl. J. Med. $308(1983), 1428$.

Dr. T. Mansky, Prof. Dr. P. C. Scriba

Klinik für Innere Medizin

Prof. Dr. H. Fassl, Dr. H.-J. Friedrich

Institut für Medizinische Statistik und Dokumentation

Medizinische Universität

2400 Lübeck, Ratzeburger Allee 160
(6) Neues - Kieler Modell- zur Krankenhausfinanzierung. Schleswig-Hostein. Arztebl., Heft 1 (1986), 57.

(7) Rhodes, R. S., C. L. Krasniak, P. K. Jones: Factors affecting length of hospital stay for femoropopliteal bypass; implications of DRGs. New Engl. J. Med. 314 (1986), 153.

(8) Smits, H. L., R. E. Watson: DRGs and the future of surgical practice. New Engl. J. Med. 311 (1984), 1612.

(9) Stern, R. S., A. M. Epstein: Institutional responses to prospective payment based on diagnosis-related groups; implications for cost, quality, and access. New Engl. J. Med. 312 (1985), 621

(10) Wenneberg, J. E., K. McPherguson, P. Caper: Will payment based on diagnosis-related groups control hospital costs? New Engl. J. Med. 311 (1984), 295.

(11) Westphal, E.: Diagnoseabhängige Fallpauschale. Dtsch. Arztebl. 82 (1985), 2616. 